Relations industrielles

Industrial Relations

\title{
Le renouvellement du régime des décrets de convention collective
}

\section{Gérard Hébert}

Volume 45, numéro 2, 1990

URI : https://id.erudit.org/iderudit/050589ar

DOI : https://doi.org/10.7202/050589ar

Aller au sommaire du numéro

\section{Éditeur(s)}

Département des relations industrielles de l'Université Laval

\section{ISSN}

0034-379X (imprimé)

1703-8138 (numérique)

Découvrir la revue

\section{Citer cet article}

Hébert, G. (1990). Le renouvellement du régime des décrets de convention collective. Relations industrielles / Industrial Relations, 45(2), 404-413.

https://doi.org/10.7202/050589ar
Résumé de l'article

Le renouvellement du régime des décrets de convention collective
Tous droits réservés (C) Département des relations industrielles de l'Universite Laval, 1990
Ce document est protégé par la loi sur le droit d'auteur. L’utilisation des services d'Érudit (y compris la reproduction) est assujettie à sa politique d'utilisation que vous pouvez consulter en ligne.

https://apropos.erudit.org/fr/usagers/politique-dutilisation/ 


\section{DISCUSSION}

\section{Le renouvellement du régime des décrets de convention collective}

\section{Gérard Hébert}

Avant de se demander s'il faut renouveler une institution, il importe de savoir si elle a une chance de survie, si les principes de fond sur lesquels elle repose sont encore valables, enfin si le renouvellement dont elle pourrait avoir besoin vise des aspects substantiels et constitutifs, ou accessoires et accidentels.

Je me propose de répondre à ces trois questions, d'abord par un coup d'oeil sur l'histoire de la loi en question, ensuite en rappelant les principes de fond qui ont amené son adoption, en 1934, et, finalement, en esquissant quelques conditions de sa survie et de son renouvellement.

\section{LA RÉPONSE DU PASSÉ}

Les périodes difficiles qu'a connues la Loi sur les décrets de convention collective (L.R.Q., c. D-2) témoignent de sa remarquable vitalité.

Un départ fulgurant et trois longues périodes de difficultés

On peut identifier trois ou quatre périodes dans la vie de la Loi relative à l'extension des conventions collectives: c'est le titre qu'elle portait lors de sa première sanction, le 20 avril 1934 (S.Q. 1934, c. 56). Elle a connu, dès le départ, un succès fulgurant. Cinq ans après son adoption, elle régissait déjà plus de 100000 salariés. Il faut se rappeler qu'il s'agissait, comme aujourd'hui encore, d'une loi volontaire, c'est-à-dire que c'est à la demande des intéressés que la loi s'applique, et non pas à l'initiative de l'État. Cinq autres années plus tard, à la fin de la guerre, c'est 200000 salariés qui étaient régis par la Loi de la convention collective (S.Q. 1940, c. 38), comme on l'a appelée de 1940 à 1964.

Pendant les quinze années du règne de Maurice Duplessis comme premier ministre du Québec,la Loi de la convention collective a connu ce qu'on pourrait appeler une période d'oubli. Le sous-ministre Gérard Tremblay, qui était responsable de son application, faisait tous ses efforts pour que le

* Hébert, G., Professeur, École de relations industrielles, Université de Montréal. 
premier ministre ne pense pas à la modifier. Malgré tout, le nombre de salariés régis n'a pas fléchi: il s'est même accru jusqu'au niveau d'environ 250000 salariés. Il s'est encore légèrement augmenté au cours des cinq premières années de la Révolution tranquille.

À compter de 1965, la Loi des décrets de convention collective a connu une période d'hostilité ouverte, au nom de la toute-puissance et de l'omniprésence de l'État-sauveur-universel. L'époque des corps intermédiaires était révolue: il fallait transférer toutes les responsabilités à l'État. Les comités paritaires perdirent, entre autres, leur autorité sur l'apprentissage et la qualification. D'un autre côté, plusieurs des parties contractantes étaient la proie de graves dissensions internes. Au bout du compte, le régime s'était passablement effrité: il avait perdu une bonne moitié de ses assujettis, tant du côté des employeurs que de celui des employés régis.

Depuis 10 ans, l'hostilité s'est poursuivie, mais pour une autre raison, au nom d'un certain néo-conservatisme qui a soufflé sur pratiquement toutes les démocraties industrialisées. L'extension juridique des conditions de travail a mauvaise presse auprès des inconditionnels de la concurrence et des lois du marché. Depuis le début des années 1980, le nombre des salariés régis par le régime des décrets a oscillé entre 125000 et 150000 .

Le régime de l'extension juridique s'est toujours maintenu malgré une hostilité quasi constante. S'il a résisté, au cours de ses 55 ans d'existence, à plus de 40 ans d'hostilité, tantôt tranquille tantôt active, c'est qu'il possède une vitalité interne qu'il n'est pas possible d'ignorer.

\section{Une survivance opiniâtre}

En un sens, le coup le plus dur qu'a dû essuyer la Loi de la convention collective, elle l'a connu en 1944, quand fut adoptée la Loi des relations ouvrières (S.Q. 1944, c. 30), copiée sur le modèle nord-américain de l'accréditation et de l'obligation légale de négocier pour les employeurs. Cette forme de relations du travail a fait passer de la loi des décrets au nouveau régime d'accréditation et de négociation des industries aussi importantes et variées que l'aluminium à Arvida, Shawinigan et Beauharnois, que la construction des wagons de chemin de fer et des fonderies de Montréal et même que l'ensemble des employés des services publics de la ville de Québec. Toutes ces entreprises ou institutions ont vu leur syndicat transférer leur convention collective de la loi des décrets à la nouvelle Loi des relations ouvrières, parce que celle-ci semblait beaucoup mieux adaptée à la situation des grandes entreprises. Par contre, la Loi sur les décrets de convention collective s'est toujours avérée utile dans les industries de forte concurrence, constituées d'un grand nombre d'employeurs, chacun avec un petit nombre d'employés; c'est dans ce type d'industries que la loi des décrets s'est concentrée presque immédiatement après l'adoption de la Loi des relations ouvrières en 1944.

Le régime a eu beaucoup plus de difficulté à se relever des durs coups subis autour des années 1970 , lorsqu'il a connu des pertes de nature fort diverse, avec l'adoption de la Loi des relations du travail dans l'industrie de 
la construction (S.Q. 1968, c. 45), mais aussi par suite de conflits internes entre les parties contractantes de certains décrets. Ces querelles intestines ont en quelque sorte provoqué le départ d'industries importantes comme la chaussure, l'imprimerie et l'alimentation au détail.

Depuis une quinzaine d'années, c'est dans le secteur des services que le régime des décrets s'est surtout développé. Il ne faudrait pas croire, d'un autre côté, que le secteur manufacturier a perdu toute son importance. Par rapport au nombre de salariés assujettis, les services représentaient, il y a quinze ans, environ $40 \%$ du nombre total des salariés régis; ils représentent aujourd'hui un peu plus de $50 \%$ de ce nombre. C'est beaucoup moins que pour l'ensemble de toute la main-d'oeuvre.

La survie des décrets et la relative stabilité du nombre de leurs assujettis - compte tenu du départ des 100000 salariés de la construction pour un régime analogue sinon semblable - ne sauraient s'expliquer, malgré toutes les oppositions persistantes au régime, sans l'appui, non pas inconditionnel évidemment, mais sans l'appui ferme et raisonné de tous les assujettis au régime, tant du côté patronal que syndical. La garantie de survie que le régime peut avoir, s'il en a une, vient de ceux qui se sont placés sous sa coupole et qui en expérimentent les avantages; le régime comporte des inconvénients bien sûr, mais les intéressés considèrent que les avantages l'emportent sur les difficultés.

D'où vient alors l'hostilité persistante qu'on a observée à son endroit? Parmi les représentants patronaux et syndicaux qui ont pris le parti de faire la guerre au régime des décrets de convention collective, on n'a peut-être pas assez souvent souligné qu'il s'agissait, presque toujours, de représentants qui n'avaient pas fait l'expérience personnelle du régime et de son fonctionnement. Très peu de représentants patronaux et syndicaux du milieu luimême ont pris une position opposée au régime; dans la plupart des cas, d'ailleurs, c'était suite à des difficultés particulières dont ils avaient été l'objet sinon la victime. Par contre, certains adversaires du régime se sont «convertis» au cours de la dernière décennie.

L'hostilité principale est venue bien davantage de certains politiciens, de quelques fonctionnaires influents, et d'un certain nombre d'intellectuels, plus près des grandes industries et des grands syndicats que des industries de concurrence, pour lesquelles le régime des décrets de convention collective est évidemment beaucoup mieux adapté que l'autre.

Les positions contradictoires que l'on observe viennent en bonne partie des principes fondamentaux sur lesquels le régime repose.

\section{LES PRINCIPES DE FOND DE L'EXTENSION JURIDIQUE}

Plusieurs des opposants au régime justifient leur position en faisant appel soit à des principes généraux, comme la suprématie de la libre concurrence ou celle de l'intervention syndicale traditionnelle, soit aux exigences du contexte économique ou syndical. Il est donc essentiel de rappeler les principes de fond sur lesquels s'appuie le régime d'extension juridique. 


\section{Un frein à la concurrence déloyable}

Les promoteurs de la loi ont toujours soutenu qu'elle avait pour objectif principal d'éliminer ce qu'ils appelaient la concurrence déloyale. La loi fut adoptée pendant la grande crise économique; tous cherchaient alors un moyen d'assurer un niveau de vie raisonnable à l'ensemble de la population, en d'autres mots un moyen de maintenir les salaires à un niveau acceptable.

Ce n'est pas par hasard que les industries qui se sont placées sous ce régime sont toutes des industries de concurrence, caractérisées par un grand nombre d'employeurs et une moyenne de salariés par employeur très faible. Celle-ci a toujours été inférieure à 10 salariés par employeur régi par la loi. Ces industries sont généralement à forte composante de travail, et le niveau des salaires y représente un élément critique pour déterminer si une entreprise sera rentable ou non, si elle survivra ou non. La concurrence sur les salaires, «sur le dos des travailleurs» comme on disait alors, est, dans ces industries, jusqu'à un certain point inévitable, à moins d'un mécanisme comme l'extension juridique. Les promoteurs du régime voulaient en quelque sorte soustraire les salaires à la concurrence et limiter celle-ci aux autres éléments du coût de production. Le travail humain n'étant pas une marchandise comme les autres, la société ne doit pas permettre que son prix (le salaire) puisse se négocier au-dessous d'un niveau raisonnable, compte tenu de la fonction occupée et de l'industrie en cause.

Ceux qui soutiennent que la concurrence doit s'exercer sans restriction sur toutes les composantes du coût de production, y compris sur les salaires, s'opposent au régime de l'extension juridique. La suppression de la concurrence sur les salaires constitue le principe de base de l'extension juridique; si on n'admet pas ce principe on ne peut que s'opposer au régime des décrets. Par contre, si on veut pousser la concurrence à ce point, on devrait être logique, et s'opposer également à tout salaire minimum au-delà de celui des pays d'où viennent les importations. Il faudrait également s'opposer à toute mesure de sécurité au travail, dès que celle-ci entraîne des coûts d'une certaine importance. Pratiquement tous les intervenants dans le débat acceptent une restriction à la concurrence; la discussion porte toujours, finalement, sur le minimum à imposer.

\section{Implication et concertation}

Un autre des principes de base du régime de l'extension juridique, d'une toute autre nature, c'est l'implication du plus grand nombre possible d'intéressés dans la détermination des conditions de travail par voie de négociation, et dans son application par le truchement du comité paritaire. La loi des décrets a permis, à chaque année de son existence, à des milliers d'employeurs et de salariés de participer de près à la détermination et à l'application des conditions de travail de leur secteur. Il y a là une forme d'implication des citoyens dans la vie économique que les initiateurs du projet avaient en vue et qui s'est effectivement réalisée. Cette forme d'implication comporte des risques: la tentation d'utiliser le régime à des fins personnelles est toujours présente. Mais ce n'est pas parce qu'une entreprise comporte 
des risques qu'on doit la délaisser. Qui, par exemple, va s'opposer à l'alphabétisation parce que plus on est instruit, plus on a de possibilités de contourner ses obligations et de s'y soustraire? Dans le cas des comités paritaires, une forme privilégiée de cette implication s'est réalisée dans le domaine de l'apprentissage et du contrôle de la qualification. Nous y reviendrons.

Comme le régime est fondé sur la négociation, la conclusion d'une convention collective et son administration par un comité paritaire, la formule implique une forme plus que minimale de concertation entre les agents socio-économiques. Ce degré de concertation a varié selon les industries et les périodes, généralement en fonction de l'engagement des principaux protagonistes, au bénéfice de leurs concitoyens et de leurs compagnons de travail ou d'industrie. À cause du rôle essentiel du gouvernement dans le processus, le régime implique une certaine forme de concertation tripartite: syndicat, patronat et gouvernement.

Peu de personnes se diront ouvertement contre la concertation. Mais plusieurs, tant du côté syndical que patronal, considèrent que la concurrence et l'affrontement donnent de meilleurs résultats qu'une coopération dont chacun des participants a peur de sortir perdant. Il y a là un dilemme fondamental: présence d'intérêts divergents et nécessité d'une coopération ou concertation, au moins minimale, faute de quoi tous risquent d'être emportés par le courant des forces extérieures, plus impitoyables que ne le sont les contraintes internes.

Pour des raisons à caractère social, les partisans du régime tiennent à ce que la concurrence se fasse en d'autres matières que sur les salaires des travailleurs. En ce sens, il est étonnant de voir certains syndicats et leurs représentants - peut-être moins aujourd'hui qu'il y a quelques années — s'opposer au régime des décrets, en disant que les travailleurs syndiqués pourraient, par les moyens de pression traditionnels, obtenir plus pour eux que dans un régime d'extension juridique; leur raisonnement implique qu'ils se désintéressent des salaires des non-syndiqués. L'effet d'osmose qu'ils invoquent à leur défense est certainement moins efficace que la présence d'un décret contraignant.

\section{L'AVENIR ET LE RENOUVELLEMENT DU RÉGIME}

Dans une perspective d'avenir, nous envisagerons les conditions de survie du régime, les domaines privilégiés de son renouvellement et les défis d'un contexte toujours changeant.

\section{Les conditions de survie}

Comme le régime repose sur une coopération bien particulière entre les agents économiques et le gouvernement, la part du gouvernement dans les conditions de survie est primordiale. Le régime est construit de telle sorte qu'il est possible de l'abolir par simple omission administrative. Il est absolument inadmissible qu'un gouvernement, par tel ou tel de ses ministres ou de ses hauts fonctionnaires, annihile la possibilité d'exercer des droits et de 
faire appliquer une loi, simplement en omettant de faire progresser les dossiers pertinents. Reporter l'adoption d'une modification pendant quelques années peut équivaloir à abolir la loi des décrets. L'abolition d'une loi ne relève ni d'un ministre, ni d'un député, encore moins des administrateurs qui sont payés pour la faire appliquer. Si une loi ne mérite plus d'exister, c'est aux élus du peuple qu'il appartient d'en décider, à personne d'autre.

Les intéressés sont évidemment en droit d'attendre davantage de leur gouvernement. Les représentants du gouvernement doivent non seulement accomplir la tâche qui leur est dévolue, mais on attend d'eux qu'ils apportent l'appui requis à un régime qui a prouvé, par ses 55 ans d'existence, qu'il répond aux attentes de plus de 100000 salariés.

De la part des intéressés, on s'attend à une participation active tant au fonctionnement du régime actuel qu'aux initiatives qu'il peut exiger et qui ne peuvent venir d'autre source que des intéressés eux-mêmes. Cette obligation ne va pas sans une certaine discipline, individuelle et collective. Coopérer comporte des avantages mais aussi des exigences: il faut oublier les avantages immédiats, souvent fort attrayants, pour assurer un avenir meilleur à plus long terme.

Enfin, il faut souhaiter que les tiers, qui n'ont pas d'intérêt direct dans les secteurs concernés, aient la politesse et la décence de ne pas profiter des pouvoirs et du prestige que leur situation peut leur conférer pour exercer des pressions en vue de modifier ou de supprimer un régime dans lequel ils n'ont bien souvent aucun intérêt direct, peut-être même pas les connaissances suffisantes pour formuler un jugement éclairé.

\section{Les domaines de renouvellement}

Le régime de l'extension juridique et des comités paritaires compte bien des aspects fort différents. Il est très important de distinguer clairement ces divers domaines; il est fort probable que les modes de renouvellement ne seront pas les mêmes dans chacun d'eux.

On peut distinguer au moins quatre domaines ou aspects, qui coexistent, mais présentent aussi un certain caractère de succession dans le temps. Premièrement, il y a la détermination des conditions de travail à extensionner, ce qui se fait par voie de négociation collective. Pour compléter cette première étape, et réaliser l'extension juridique des conditions négociées, le gouvernement intervient pour évaluer la demande soumise, permettre aux tiers intéressés de faire connaître leur point de vue et décider de l'adoption ou non du décret. Cette intervention gouvernementale fait partie du régime lui-même; elle réalise la concertation tripartite qui est la clef de voûte de tout le système.

En second lieu, vient l'application et la surveillance des conditions déterminées et décrétées à l'étape précédente. Selon la loi telle qu'elle existe depuis ses débuts, c'est le comité paritaire qui est responsable de cette partie, de ce domaine spécifique du régime. 
Le troisième domaine où le régime a excellé, tout spécialement dans les années 1950 et 1960, c'est celui de la formation et de la qualification professionnelles. Un amendement de 1946 à la Loi de la convention collective permettait aux comités paritaires de consacrer des fonds à l'apprentissage. Un grand nombre d'entre eux l'ont fait, et généralement avec beaucoup de succès. En 1969, la Loi des décrets de convention collective a été amputée de sa section sur l'apprentissage, au profit du ministère de l'Éducation et plus tard du Travail et de la Main-d'oeuvre, par le truchement des Commissions de formation professionnelle. Sans porter de jugement sur l'efficacité des nouveaux organismes, on peut dire que le fruit de nombreuses années d'efforts de la part de plusieurs comités paritaires s'est dissipé en paroles et en papier sinon en fumée. Quelques comités paritaires se prévalent encore aujourd'hui des mesures transitoires de 1969 et interviennent en matière de qualification professionnelle; certains s'interrogent sur la valeur d'une mesure transitoire vingt ans après son adoption.

Il faudrait mentionner un quatrième domaine où il y a eu quelques tentatives d'amorcées; ce domaine fourmille de possibilités, mais il compte encore bien peu de réalisations. Il s'agit de l'observation prospective et de l'orientation des secteurs économiques; les comités paritaires concernés pourraient s'adonner à des activités inappréciables en la matière. À ce niveau, la concertation et la coopération s'avèrent indispensables dans le contexte actuel.

Notons que les deux premiers domaines, celui de la négociation et de l'application - nous reviendrons plus loin sur les deux autres - exigent peut-être des adaptations, mais l'expérience des 50 dernières années montre que l'instrument est passablement rodé et à point, du moins dans sa structure d'ensemble. Ce qui ne veut pas dire qu'il n'y a pas des points à améliorer. À titre d'exemples mentionnons les suivants. Certains comités paritaires n'ont pas toujours été sans reproche dans leur surveillance du décret. Certains cas de «compromis jugés convenables», prévus par la loi, ont pu frôler le pur favoritisme. La surveillance gouvernementale en la matière a pu manquer de rigueur à tel ou tel moment.

Plus sérieuse est la question du règlement des plaintes. Bon an mal an, les comités paritaires récupèrent deux ou trois millions de dollars des employeurs délinquants pour les remettre aux salariés visés. C'est un aspect qu'il ne faut ni oublier, ni négliger. Mais certains regrettent que le régime ne favorise pas un mode de règlement des plaintes qui englobe d'autres griefs que les pertes monétaires. Ils souhaitent une plus grande implication des représentants syndicaux, actuellement relégués à un rôle de moindre importance. Sans oublier la possibilité, qui existe toujours, de négocier des conventions collectives complètes avec les employeurs dont les employés sont syndiqués, il faut dire que le problème, dans la mesure où il rejoint les ateliers non syndiqués, est bien réel et qu'il mérite qu'on s'efforce de lui trouver une solution satisfaisante.

Mais les difficultés d'un régime ne doivent pas nous faire oublier ses avantages réels. Il faut chercher à résoudre les problèmes sans détruire le régime lui-même. Dans cette perspective, nous tournons notre attention vers les deux autres domaines mentionnés, celui de la formation et de l'orientation des secteurs. 


\section{Le contexte présent et les défis qu'il pose}

Parler du contexte présent, c'est évoquer le libre-échange, la mondialisation des marchés, les changements technologiques qu'on introduit à un rythme accéléré. Les adversaires du régime des décrets le considèrent comme un organisme figé, incapable de faire face aux exigences nouvelles du moment présent. Bien au contraire, le régime de l'extension juridique et des comités paritaires paraît tout à fait bien adapté à la situation qu'on peut prévoir dans les prochaines décennies.

Dès qu'on aborde la question de l'adaptation de notre économie au contexte futur, le mot qui revient sur toutes les lèvres, c'est celui de la formation de la main-d'oeuvre. En cette matière, au cours des 50 dernières années, les expériences les plus valables et les plus performantes ont été celles qui furent mises de l'avant et soutenues par des comités paritaires. Les expériences de formation et de qualification professionnelles ne sauraient réussir si elles se poursuivent loin des premiers intéressés, c'est-à-dire des employeurs et des représentants des travailleurs concernés, à la base. Le mythe de l'État-sauveur-de-tous-les-défavorisés est bel et bien dissipé. L'échec de la plupart des prévisions et planifications de main-d'oeuvre est aussi un phénomène généralement reconnu et acquis. C'est vers la base qu'il faut se retourner pour établir quels seront, à moyen terme, les besoins de main-d'oeuvre, et quels sont les moyens les plus efficaces à adopter pour y faire face.

Il serait éminemment souhaitable que cet effort soit fait dans tous les secteurs où existent des comités paritaires, avec l'appui du gouvernement, par exemple en redonnant aux intéressés un certain pouvoir en ce domaine. Par contre, il faut être réaliste et ne pas espérer que les querelles de clocher entre les différents ministères et fonctionnaires vont $s^{\prime}$ estomper dans un avenir prochain. Comme citoyen et observateur de cette réalité, $j$ 'ai beaucoup plus confiance aux initiatives que pourront prendre les parties intéressées dans la matière. Faute de l'appui de l'État, les parties intéressées peuvent réaliser énormément, en établissant un consensus entre elles et en posant les premiers jalons d'une véritable politique de main-d'oeuvre dans leur secteur respectif. Quand les parties intéressées auront fait les concessions requises, l'une envers l'autre, pour arriver à cette fin, le gouvernement n'aura d'autre possibilité que d'emboîter le pas et de suivre ceux qui lui auront tracé le chemin.

Au niveau des secteurs, compte tenu du contexte actuel et de ce qu'on peut prévoir pour les années à venir, la concertation entre les agents économiques - et le gouvernement si possible - s'avérera une nécessité. Il faudra des organismes qui surveillent constamment l'état de santé de chaque secteur industriel, qui trouvent les solutions appropriées aux problèmes soulevés et qui aident à leur réalisation. Les premiers secteurs concernés sont évidemment ceux de la production; à ce sujet; il ne faut pas oublier que l'avenir ne semble pas aux très grandes entreprises, mais à celles de petite et de moyenne dimension, où le régime de l'extension des conventions collectives s'applique particulièrement bien. 
Certains comités paritaires ont amorcé des réflexions de cette nature. Il faudrait qu'ils intensifient leurs efforts en ce sens, et que d'autres emboîtent le pas, pour qu'éventuellement cette fonction leur soit reconnue of ficiellement. Aucun organisme n'est mieux placé que le comité paritaire pour remplir cette fonction d'observateur et d'orienteur des secteurs concernés. Et que les secteurs de services ne se considèrent pas à l'abri de l'évolution technologique et concurrentielle du monde. Rien ne nous laisse croire que l'entretien des édifices, la sécurité des lieux ou même la collecte des ordures ménagères ne subiront pas le contrecoup de l'évolution technologique et mondiale: qu'on pense simplement aux problèmes des contaminants et de l'environnement. Il ne s'agit pas de s'engager dans des études à l'envergure du monde. Tout ce que l'exercice requiert des intéressés, c'est de regarder au-delà de leurs intérêts immédiats et particuliers pour, en quelque sorte, voir venir le monde et ses changements futurs. L'exercice exigera très certainement l'abandon de quelques avantages immédiats et l'investissement dans des études dont on ne voit pas toujours les résultats prochains; mais la survie de chaque secteur concerné, pour ne pas dire notre survie à tous, l'exige impérieusement.

L'évocation de cette possibilité peut paraître futuriste. Mais outre la formation et la qualification professionnelles, ce domaine d'investigation et de prospective de la part des comités paritaires apparaît comme l'épanouissement normal de ce qu'ils ont vécu depuis leur établissement, malgré l'hostilité qu'ils ont subie de le part de bien des adversaires. C'est là ma réponse à la question posée: un tel renouvellement des comités paritaires me semble une nécessité, pour leur survie, pour leur progrès et pour celui de toute notre société.

\section{Updating the Collective Agreement Decree System}

Does the system have a future? Of what kind and under what conditions?

\section{LESSONS FROM THE PAST}

After a dazzling growth period (1934-1944), the decree system was happily forgotten during the Duplessis era; this ensured good working conditions every year of that period to over 200000 workers. Since the mid-60s, the decree system came under attack by the all-pervasive-State advocates and was simultaneously undermined by severe internal divisions: its coverage fell by half to 125000 . From 1980 and still today, it is one of the prime targets of the neo-conservatism and staunch defenders of unrestricted competition and the market mechanism.

The decree system has survived all these attacks, surely because it is a useful form of labour relations in the industrial and service sectors in which it has developed. Maybe also because its fundamental tenets successfully answer a major social problem in the highly competitive sectors where it applies. 


\section{FUNDAMENTAL TENETS}

The system has been devised to eliminate wage competition and thus protect both the workers by ensuring reasonable wages and the employers willing to pay decent wages against unfair competition from unscrupulous employers.

Through the joint and parity committees, the system has brought thousands of employers and workers to take an active part in critical socio-economic decisions for their industry, and in a unique experiment of cooperation and tripartism (contracting parties and government).

\section{CHALLENGES OF THE FUTURE}

Conditions of survival - First and foremost, the government must discharge its duties mentioned in the law. It is unacceptable that a law be in practice abrogated by anybody else than by the duly-elected House members.

The other condition rests with the interested parties: they must take an active part in the operation of the law, at all stages, and bear its disadvantages as well as enjoy its advantages.

Participation stages - The process can be broken into four stages. The first two have been in satisfactory operation for over 50 years: the bargaining by the parties of proper decree provisions and their policing by the joint committee.

Despite some difficulties, apprenticeship and qualification control have been the finest jewels of the system from 1945 to 1969 . The all-powerful State that emerged from the Quiet Revolution of the 60s took away that responsibility from the joint committees unto itself.

A new domain of responsibility, at a higher level, could open up for the joint committees: an on-going assessment of the industrial sector, its current position, its problems, and the ways and means to solve them.

Current challenges - In the present context of free-trade, world markets and increasing velocity of technological change, the one and universal remedy proposed is workers' training and retraining. No institution is better prepared to handle this program than the joint committees.

To ensure the best on-going assessment of an industrial sector and the most efficient long-term solutions to its problems, the best locus of discussion and cooperation is also with the joint committees where they exist.

If government offers these committees the proper aid, so much the better. If not, it will be the responsibility of the interested parties to take the required initiatives. The government afterwards will have no other choice than to follow suit. 